\title{
NUEVA ESPECIE DEL GÉNERO LESTES LEACH, 1815 (INSECTA, ODONATA) DEL MIOCENO DE BELLVER DE CERDANYA (LÉRIDA)
}

\author{
Arturo Compte-Sart* \\ * Museo Nacional de Ciencias Naturales. Departamento de Biodiversidad y Biología Evolutiva. Madrid. España. \\ Dirección postal: c/. Rafaela Bonilla 19, escalera J, $1^{\circ}$ D. 28028 Madrid. España \\ urn:Isid:zoobank.org:author:EED96568-6539-481A-B736-77A10E90113E
}

\section{RESUMEN}

Se describe la nueva especie fósil de insecto Lestes dianacompteae n. sp. del orden Odonata, perteneciente al género Lestes Leach, 1815, sobre un ala procedente del yacimiento de Coll de Saig, en Bellver de Cerdanya (Lérida, España), del piso Vallesiense (Mioceno). La especie es comparada con todas las fósiles del género Lestes y las actuales más parecidas de las regiones Paleártica y Etiópica.

urn:Isid:zoobank.org:pub:0E5AC6D1-525D-46B8-AA38-DFDD2D94D337

Palabras clave: Odonatos; Lestes fósiles y actuales; Lestes dianacompteae n. sp.; fósil; Vallesiense; Mioceno; Bellver de Cerdanya; Lérida (España).

\section{ABSTRACT}

A new species of genus Lestes Leach, 1815 (Insecta, Odonata) from Miocene of Bellver de Cerdanya (Lérida)

A new fossil species of insect, Lestes dianacompteae, n. sp. of the order Odonata, belonging to the genus Lestes Leach, 1815, is described based on wing from the Vallesian (Miocene), of Coll de Saig, in Bellver de Cerdanya (Lérida, Spain). It is compared with all fossil species of the genus and with the nearest extant species, from the paleartic and ethiopian regions.

Keywords: Odonata; fossil and extant Lestes; Lestes dianacompteae n. sp.; fossil; Vallesian; Miocene; Bellver de Cerdanya; Lérida (Spain).

Recibido/Received: 15/05/2013; Aceptado/Accepted: 16/01/2014; Publicado en línea/Published online: 1/08/2014

Como citar este artículo/Citation: Compte-Sart, A., 2014. Nueva especie del género Lestes Leach, 1815 (Insecta, Odonata) del Mioceno de Bellver de Cerdanya (Lérida). Grael/sia, 70(1): e001. http://dx.doi.org/10.3989/graellsia.2014.v70.097

Copyright: (C) 2014 SAM y CSIC. Salvo indicación contraria, todos los contenidos de la edición electrónica de Grael/sia se distribuyen bajo licencia de uso y distribución Creative Commons Reconocimiento no Comercial 3.0. España (cc-by-nc).

\section{Introducción}

Hace muchos años me presentaron el fósil mencionado en el título de este trabajo, en consulta para una publicación sobre insectos del Mioceno de la Cerdanya (Lérida). Confirmado el género y que la especie era nueva para la ciencia, sugerí que fuera nominada pero prefirieron mencionarlo solamente como Lestes sp. (Arillo \& Sanz de Bremond, 1992).
Como el estudio realizado había sido muy laborioso creí conveniente aprovecharlo para publicarlo a mi vez, dando nombre al insecto y de paso hacer una reseña del conjunto paleontológico. La extensa descripción fue calificada muy negativamente por un revisor, que estimó además que no estaban adecuadamente detallados los aspectos taxonómicos de la nueva especie. En consecuencia retiré el trabajo para una posible revisión futura. 
En los más de 20 años transcurridos se han hallado al menos otras 7 especies de Odonatos fósiles en la cuenca ceretana pero nadie ha intentado nominar el léstido que nos ocupa. Por este motivo he retomado la descripción, avalada por la extensa bibliografía consultada y la gran cantidad de material de comparación utilizado. Conviene añadir que entre las muchas publicaciones recientes sobre geología y paleontología de la comarca destacan las de insectos (Nel et al., 1996; Peñalver et al., 1999) y una importante tesis doctoral sobre la flora miocena de la Cerdanya (Barrón, 1996), todo lo cual enriquece el conocimiento del ecosistema del Mioceno superior ceretano.

La geología y estratigrafía de la depresión de la Cerdanya están descritas como conjunto por Vera (2004) y en detalle por Agustí \& Roca (1987), Cabrera et al. (1998) y Anadón et al. (1989). El fósil que se estudia procede del yacimiento de Coll de Saig, descrito en numerosas publicaciones, una recopilación de las cuales figura en la tesis doctoral, inédita, de Barrón (1996) y en el resumen sobre Coll de Saig del mismo autor (1999).

La depresión de la Cerdanya es el resultado de una importante falla asociada a otras menores que ocasionaron entre otras, la fosa neógena de aquel nombre, que a lo largo del Mioceno superior fue rellenándose con potentes sucesiones detríticas, estructuradas en dos grandes unidades estratigráficas: la Unidad Neógena Inferior, de potencia variable de 400 a 800 m (desarrollada a lo largo del Vallesiense y gran parte del Turoliense), y la Unidad Neógena Superior, de potencia de unos $250 \mathrm{~m}$ (iniciada en el Turoliense superior, culmina la colmatación de la cuenca en el Plioceno). La cuenca lacustre formada tiene actualmente unos 30 $\mathrm{km}$ de largo y de 5 a 7 de ancho y una altitud media de $1100 \mathrm{~m}$; en su margen septentrional está bordeada de altas montañas de unos $3000 \mathrm{~m}$ y en el meridional, la Sierra del Cadí, con alturas en torno a $2500 \mathrm{~m}$, formándose en su día un gran lago mioceno relativamente profundo hacia el SO de la cuenca. El yacimiento de Coll de Saig debe corresponder a sedimentos cercanos a la orilla septentrional del antiguo lago. Pertenece, en la nomenclatura estratigráfica de Anadón et al. (1989), al tramo C, de diatomitas y cienos grises, de la Unidad Neógena Inferior. El yacimiento se abre por una trinchera en una loma o collado, que fue practicada para el trazado de la carretera comarcal 1411, de Bellver de Cerdanya a Prats, a un $\mathrm{km}$ de éste y a unos $1120 \mathrm{~m}$ de altitud, con una potencia de 2-1,5 $\mathrm{m}$.

Hay numerosos estudios paleoambientales de Coll de Saig, aunque a veces contradictorios. Según Menéndez Amor (1955), dominaba la flora forestal caducifolia, muy variada; las lluvias eran abundantes todo el año, causando un ambiente húmedo, con veranos cálidos y largos e inviernos moderadamente fríos, con temperaturas medias anuales de unos $15^{\circ} \mathrm{C}$, los de enero de $6,5^{\circ} \mathrm{C}$ y los de julio de $24^{\circ} \mathrm{C}$. Álvarez Ramis y Golpe Posse (1982), consideran que la flora era principalmente de tipo templado o intertropical, con escasos elementos tropicales, de ambientes secos en zonas elevadas y vertientes alejadas de la humedad del lago, pero en el área lagunar y fluvial habría alta humedad. Barrón (1996, 1999), señala que en la zona había extensos bosques mixtos mesofíticos, laurisilvas y formaciones riparias, con bosque mixto de coníferas en las zonas más altas; el clima debía ser templado cálido o subtropical, con abundantes lluvias y alta humedad ambiental. Barrón (1999), opina que Coll de Saig probablemente corresponde a una zona lacustre alejada de la orilla, contrariamente a otro autor (Martín Closes, 1995), que creía que era una zona de la ribera, en donde se acumulaban los restos vegetales transportados por vientos y corrientes. Margalef (1957), ciñéndose a la supuesta evolución del lago, de la oligotrofia a la eutrofia, pero no a su desecación (ya que estima la profundidad en torno a $10 \mathrm{~m}$ ), considera que el clima era muy cálido pasando a una etapa bastante fría y lluviosa, con una temperatura que podría ser de 15 a $18{ }^{\circ} \mathrm{C}$. Puede añadirse que los insectos fósiles hallados no permiten aventurar datos ambientales, excepto quizá los Odonatos Stenolestes hispanicus y Trithemis pseudodistanti, que son géneros que vivieron y viven en climas subtropicales o claramente tropicales; el último se desarrolla en aguas corrientes o estancadas. El género Lestes es cosmopolita y de muy diversos ambientes, templado-fríos a tropicales, desarrollándose en aguas estancadas o de corriente débil.

\section{Material y métodos}

Se trata de la compresión de un ala de Odonato casi completa. Se conservan venas y celdas como restos carbonosos en buen estado, excepto el ápice alar, algo del pterostigma y un breve segmento marginal en la base alar posterior. Además las principales venas cóncavas, como la subcostal posterior, los sectores de la radial y la media posterior, están bastante erosionadas, pero su trazado y las celdillas pueden reconocerse en su mayor parte con iluminación oblicua, permitiendo casi siempre reconstruirlas con bastante exactitud.

El ejemplar forma parte de los numerosos fósiles recogidos por los paleontólogos Villalta y Crusafont hacia 1943, en la depresión miocena de Bellver de Cerdanya (Lérida), reseñado en su publicación de 1944.

La roca matriz del fósil es una plaquita de $55 \mathrm{~mm}$ por $25 \mathrm{~mm}$ y $15-10 \mathrm{~mm}$ de grueso. Aspecto de fino cieno, gris pálido o blanquecino, algo untuoso al tacto, suave y fácilmente disgregable. Contiene algunos fragmentos muy pequeños líticos y otros oscuros mayores, restos de vegetales mineralizados, además de muy pequeños núcleos de óxido de hierro.

La pieza tiene en un ángulo del anverso las iniciales C. S. escritas a lápiz, y en un lateral en tinta negra el número ${ }^{\vee} 9859$ sobre fondo de pintura blanca. 
En la bolsita de plástico que lo contiene hay una etiqueta suelta impresa, de 75 por $36 \mathrm{~mm}$, que dice "Ajuntament de Barcelona, Museu de Geología, Secció Paleontología. Núm. "9859 col. Villalta". Hay añadido en tinta manuscrita la indicación: "25/3/88 J. Gómez-Alba. Ala de Neuróptero. Mioceno sup. Badés, Cerdanya (sin número ni etiqueta original, porta la sigla C. S.: Coll de Saig)".

La publicación de los colectores Villalta y Crusafont (1944: pág. 353 y lám. 6), sólo dice "Neuróptero" en el texto y en la fotografía "Ala de Neuróptero. Badés". En la imagen falta la indicación "C. S.", que existe ahora en la placa del fósil, por lo que es evidente que fue añadida posteriormente, quizá para rectificar un error de asignación en la fotografía primera. Esto puede plantear la duda de cuál es el verdadero yacimiento de procedencia, aunque aquí se adopta el nombre de Coll de Saig por creerlo más probable. Esto aparte, conviene añadir que Coll de Saig y Badés están topográficamente próximos y sin duda son de parecidos niveles estratigráficos, ya que las biozonas son similares, ricas en restos vegetales e insectos, y las características físico-químicas de sus sedimentos son también parecidas pero las margas de Badés tienen mayor proporción de arcilla, hierro y magnesio, con menos calcio (Rodríguez Mellado y Menéndez Amor, 1948: 69-71), y son algo más oscuras y bastante más duras, dificultando un poco la extracción de fósiles (Cerezo y Vilario, 1947: 576). Margalef (1957) sitúa estratigráficamente muy próximos ambos yacimientos, siendo Badés el inferior.

Cabe añadir que la citada fotografía muestra el ala en muy buen estado, prácticamente completa, por lo que posteriormente debió haber algún deterioro, seguramente por frotación, debido a la fragilidad de los restos fosilizados. La destrucción de parte de las venas cóncavas, que en la placa deberían haber sido las mejor protegidas, puede que se deba a que hubieran quedado adheridas a la desaparecida contraplaca.

Se ha intentado precisar si es un ala anterior o posterior, porque en los Lestinae (aunque en menor grado que en los Sympecmatinae), ambas alas generalmente no son idénticas. Comparando numerosas especies resulta que el lado basal del cuadrilátero discoidal con frecuencia es igual o mayor que el costal en las anteriores y algo menor en las posteriores. Como en el fósil el lado basal es claramente más largo que el costal se considera muy probable que se trate de un ala anterior.

La identificación de la familia se ha basado en las obras de Fraser (1957), Pinhey (1962), Davies (1981) y Bechly (1998), resultando evidenciado que se trata de la familia Lestidae.

La complicada delimitación y determinación de los géneros y subgéneros considerados se ha realizado con las obras de Selys (1862), Munz (1919), Fraser (1933, 1957), Lieftinck (1960), Schmidt (1929, 1966), Pinhey (1980), Watson et al. (1991) y Nel \& Paicheler (1994).
Para el catálogo de especies fósiles, además de las obras de Kirby (1890), Handlirsch (1906-1908) y Carpenter (1992), se ha empleado principalmente la obra de Bridges (1993), con las adiciones de Nel et al. (1994, 1997), Peñalver et al. (1996) y la compilación crítica sistemática de Nel \& Paicheler (1994).

En el estudio de las 20 especies fósiles atribuidas al género Lestes, se han utilizado principalmente las descripciones originales de todas ellas, excepto la de Lestes zalesskyi Piton, 1940, cuyo examen se ha basado en la descripción de Nel \& Paicheler (1994: 7). La revisión de estos últimos autores ha sido de gran ayuda para completar las descripciones o figuras de varias especies.

Aunque es poco probable que una especie del periodo mioceno, de hace diez millones de años, pudiera vivir todavía, se ha creído conveniente revisar las especies actuales de Lestidae. Sin embargo esto sólo es posible comparando las alas, de ejemplares de museo o iconografía, lo cual a nivel mundial es impracticable porque la mayoría de especies están descritas sin diseños alares y las monografías carecen de dibujos (Selys, 1862; Martín, 1910) o son insuficientes (Pinhey, 1980) teniendo en cuenta que la familia es cosmopolita y tiene más de 140 especies (Bridges, 1993).

Como señala críticamente Lieftinck (1960), la variabilidad interespecífica de los caracteres utilizados, incluso en larvas, y el dudoso valor de algunos de ellos, como es el color del cuerpo o de las alas, hacen discutibles o de límites imprecisos muchas divisiones genéricas, por lo que el número de géneros $\mathrm{y}$ subgéneros es muy variable según sean los autores: 15 géneros (Fraser, 1951), 12 géneros y 6 subgéneros (Davies, 1981), 8 géneros y 7 subgéneros (Bridges, 1993), etc. siendo el criterio de éste último autor el adoptado aquí.

En consecuencia, se ha considerado conveniente limitar el estudio comparativo a las especies actuales de Lestidae de las regiones Paleártica y Etiópica, como más probables áreas en las que podrían hallarse las especies más parecidas al fósil en estudio. Por ello se ha utilizado material de museo y la iconografía y datos de las descripciones (tamaño, pterostigma, venillas postnodales, celda discoidal, etc.), que permitan suficientes comparaciones. Se ha dedicado especial atención a los subgéneros Lestes s. str., Chalcolestes Kennedy, 1920 y Pesudochalcolestes Pinhey, 1980 (unas 20 especies), utilizando las publicaciones de Selys (1883), Ris (1921), Schmidt (1929, 1951), Needham (1930), Pinhey (1962, 1980), Belyshev (1973) y Askew (2004).

Los Catálogos generales utilizados de especies actuales han sido los de Davies (1981), Davies \& Tobin (1984) y Bridges (1993).

Kennedy (1920: 84), describió el género Chalcolestes para $L$. viridis V. d. Lind., diciendo que difiere de Lestes porque el segmento superior del arculus es igual al segmento inferior. En realidad se refería a que 
la vena radial posterior incide en medio del arculus anterior, en lugar de cerca de la celda discoidal, como ocurre en $L$. sponsa Hans., especie tipo del género Lestes. Posteriormente Fraser (1951: 68), caracteriza Chalcolestes por tener la vena anal anterior separada de la posterior (borde alar), antes de la venilla transversal de la cubital posterior. Otros autores añaden que la larva tiene una morfología diferente de los demás Lestes (sobre todo europeos). Pseudochalcolestes Pinhey (1980: 355) difiere entre otros caracteres, por la vena radial posterior próxima a la celda discoidal. Ambos taxones se consideran aquí subgéneros de Lestes.

La datación del fósil se estima con relativa aproximación ya que no se conoce el horizonte preciso del que procede. Coll de Saig ha sido datado como Turoliense o tal vez Plioceno inferior (Rodríguez Mellado y Menéndez Amor, 1948); primeramente de la base del Mioceno superior, posteriormente de horizontes del tramo alto (Margalef, 1957); Vallesiense-Turoliense (Álvarez-Ramis y Golpe-Posse, 1982) y Vallesiense medio-superior (Roca, 1986, in Barrón 1999). Aquí se adopta la determinación de Vallesiense medio-superior de los últimos autores citados.

La cronología estratigráfica de Coll de Saig se establece entre 11,5 y 9,2 millones de años, según el importante estudio de Calvo et al. (1993), que en líneas generales coincide con las publicaciones más recientes de Vera (2004) y Ogg (2004).

Para la nomenclatura de la venación alar se ha seguido la propuesta por Riek y Kukalová-Peck (1984), con algunas modificaciones de Bechly (1998). Como la notación consta en las figuras 2 y 4 de la descripción, no hay dificultad en compararla con las denominaciones venales de otros autores.

El estudio se ha realizado con un microscopio estereoscópico Zeiss con aumentos básicos de 6 a 40, de iluminación acoplada, retículo ocular milimétrico y cámara clara para dibujo, de gran nitidez. Las fotografías, de cámara especial acoplada, son de alto contraste, tomadas en varios ángulos de incidencia luminosa. Las mediciones se han efectuado combinando el retículo milimétrico con las fotografías ampliadas, con escala milimétrica y uso del compás de puntas.

\section{Lestes dianacompteae nov. sp.}

Material estudiado: Un ala casi completa, muy probablemente del par anterior (figs. 1-2). Etiquetada con el número 9859 colección Villalta, del Museo de Geología, Sección de Paleontología, de Barcelona.

Localidad: Coll de Saig, en Bellver de Cerdanya (Lérida, España).

ÉPOCA: Mioceno superior, piso Vallesiense medio-superior, Unidad Neógena Inferior, tramo C, estrato de cienos gris pálido.

EDAD: Entre 11,5 y 9,2 millones de años.

Diagnosis: Tamaño mediano: longitud del ala $22 \mathrm{~mm}$; relativamente ancha, $4,75 \mathrm{~mm}$. 12 venillas postnodales. Pterostigma casi triple largo que ancho:
$1,75 \mathrm{~mm}$ por $0,62 \mathrm{~mm}$, cubriendo dos celdas. Venas $\mathrm{IR}_{2}$ y $\mathrm{RP}_{3-4}$ bien separadas, por $0,57 \mathrm{~mm}$, casi la mitad de la distancia al arculus y 4,4 veces distantes del subnodo. Celda discoidal ancha: lado basal 0,52 $\mathrm{mm}$, mayor que el costal, de $0,42 \mathrm{~mm}$, y poco más de 2,5 veces el inferior, de $1,37 \mathrm{~mm}$. Venilla transversa CuP situada poco antes de la mitad de las dos antenodales. La RP sale de la base del arculus superior. Dos hileras de celdas entre MP y AP.

DESCRIPCIÓN: El contorno alar es casi completo, con la excepción de la mitad distal del borde costal, que está fragmentado, algo del ápice alar (cuya huella se distingue en la impresión del molde arcilloso), y un breve segmento en la base del ala. También faltan en parte varias venas longitudinales cóncavas, pero su trazado puede seguirse con relativa facilidad, lo mismo que en las venillas transversas correspondientes, que limitan las celdillas. Las venas y venillas son de color pardo oscuro o castaño negruzco. El pterostigma es gris algo oscuro. No existen manchas discales.

Longitud total del ala $21,6 \mathrm{~mm}$; incluyendo el fragmento apical que falta tiene algo más de $22 \mathrm{~mm}$. Forma alar relativamente ancha, con la mayor amplitud algo después de los dos tercios basales. Anchura máxima $4,75 \mathrm{~mm}$ (a la altura de la $8^{\mathrm{a}}$ venilla postnodal); anchura al nivel del nodo 3,12 $\mathrm{mm}$.

Longitud del pedúnculo aproximadamente $3 \mathrm{~mm}$; anchura al nivel de la venilla transversa $\mathrm{CuP}, 1,30 \mathrm{~mm}$.

Pterostigma (un poco erosionado), 1,75 $\mathrm{mm}$ de largo y $0,62 \mathrm{~mm}$ de ancho. Cubre dos celdillas, aunque supera algo la distal. La venilla proximal del pterostigma (de refuerzo o abrazadora), está casi entera pero su prolongación en el campo subcostal (en realidad, el campo es de la radial anterior), está rota por erosión.

Hay dos venillas antenodales. La primera está situada a unos 2,25 $\mathrm{mm}$ de la base alar, mucho antes que la transversal anal CuP, y ésta se encuentra ligeramente antes de la mitad de la distancia entre la $1^{\mathrm{a}}$ y la $2^{\mathrm{a}}$ antenodales. Se observan 12 venillas postnodales.

La distancia entre las antenodales es de 1,46 mm. De la $2^{\mathrm{a}}$ antenodal al nodo hay $3,60 \mathrm{~mm}$.

La $2^{\mathrm{a}}$ antenodal prolongada en la subcostal incide en la base del arculus (arquillo), que es la rama inferior (RP) de la primera bifurcación de la R. De este punto de la RP al subnodo hay $4,18 \mathrm{~mm}$. Las venas $\mathrm{RP}_{3-4} \mathrm{y}$ $\mathrm{IR}_{2}$ comienzan relativamente cerca del arculus y están moderadamente separadas entre sí $(0,57 \mathrm{~mm})$. La distancia del arculus a la $\mathrm{RP}_{3-4}$ es de $1,10 \mathrm{~mm}$. Entre la $\mathrm{IR}_{2}$ y el ápice de la subnodal hay $2,51 \mathrm{~mm}$.

El nodo es bastante grueso, con la rama superior (parte superpuesta de la $\mathrm{ScP}$ ) en triángulo irregular y la inferior (mitad de la vena nodal, entre la ScP y la RA), muy ancha y con un pequeño orificio central, en óvalo alargado, probablemente por haberse partido la vena al estar presionada; su prolongación, la venilla transversa subnodal, entre la RA y la RP, es simple y entera. 


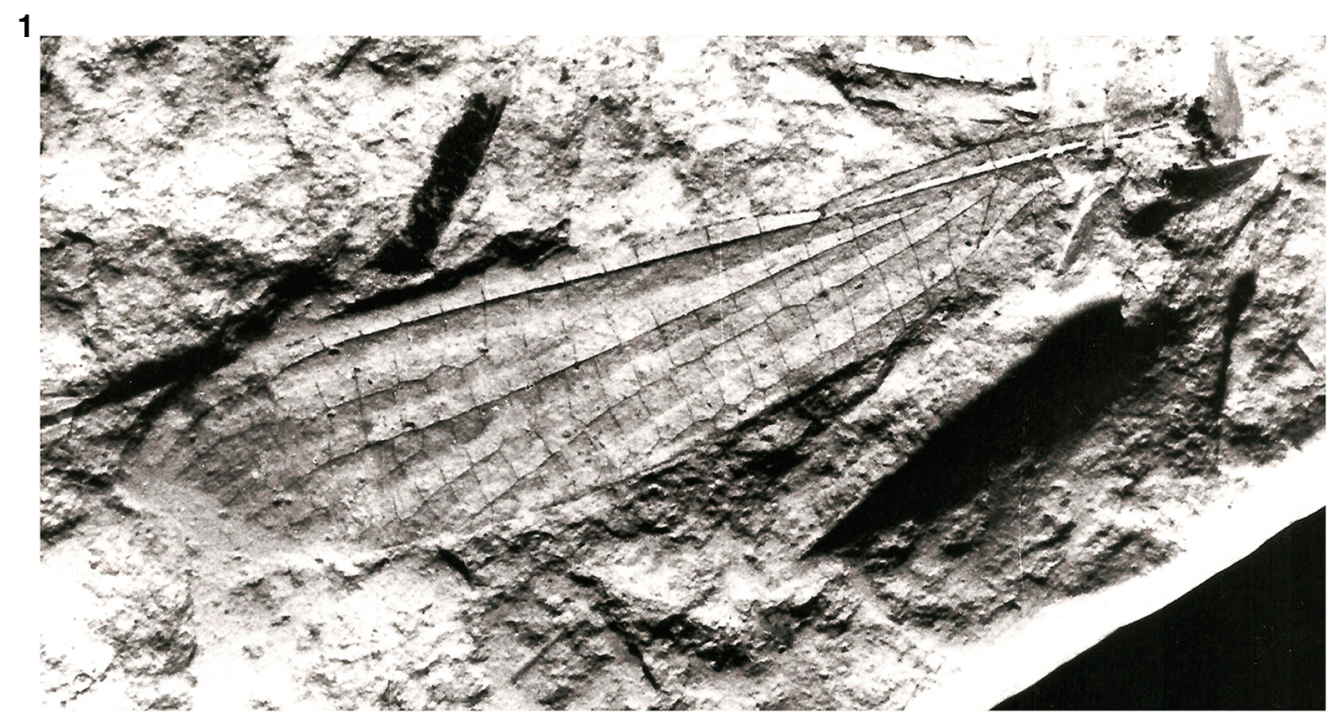

2

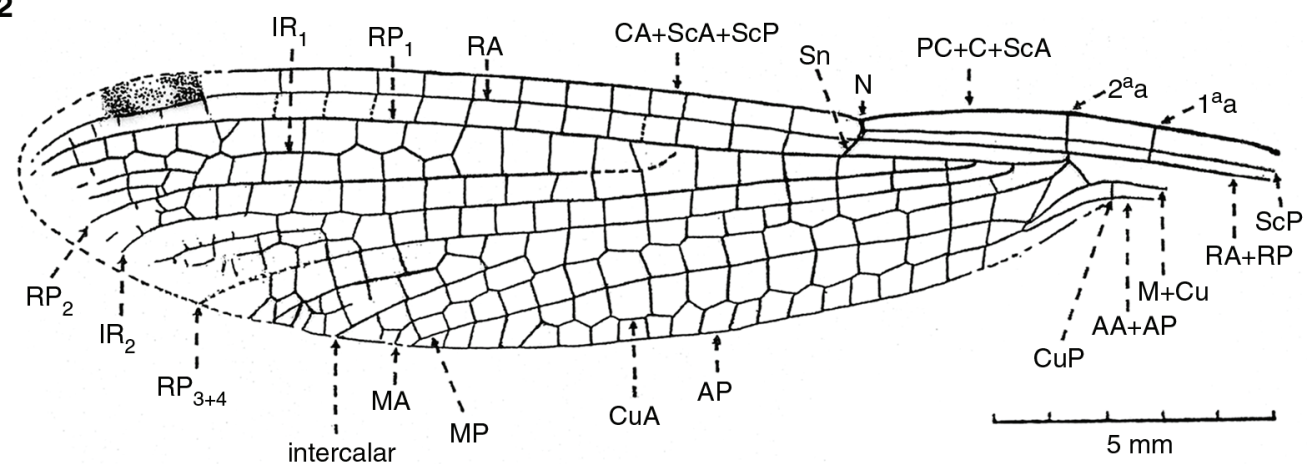

Figs. 1-2.- Lestes dianacompteae n. sp. 1) Ala entera, 2) Diseño venal.

Figs. 1-2.- Lestes dianacompteae n. sp. 1) Complete wing, 2) Venal design.

De la base alar al nodo hay $7,37 \mathrm{~mm}$; de éste al pterostigma $11,61 \mathrm{~mm}$ y del nodo al ápice alar aproximadamente $15 \mathrm{~mm}$.

El cuadrilátero discoidal (que en realidad debería denominarse discal), es ancho, casi triangular, con el lado basal o interno de $0,52 \mathrm{~mm}$, claramente mayor que el lado superior o costal, de $0,42 \mathrm{~mm}$. El lado distal mide $0,93 \mathrm{~mm}$ y el lado inferior $1,37 \mathrm{~mm}$. El subcuadrilátero es estrecho, levemente curvado y con el ápice distal oblicuamente truncado (figs. 3-4).

La venilla transversa $\mathrm{CuP}$ está situada a $0,36 \mathrm{~mm}$ del cuadrilátero discoidal, siendo bastante mayor el lado basal de éste $(0,52 \mathrm{~mm})$.

La vena $R$ se divide aparentemente en anterior y posterior muy separadamente, tanto como la longitud del arculus anterior. La $\mathrm{RP}_{2}$ parece separarse de la $\mathrm{RP}_{1}$ a la altura de la tercera venilla postnodal, mientras que la $I R_{1}$ sale a la altura de la postnodal $8^{\mathrm{a}}$. La venilla oblicua es la tercera transversa después del arranque de la $\mathrm{RP}_{2}$ y está al nivel de la $6^{\mathrm{a}}$ postnodal.

La separación de la AA y la AP no puede observarse debido a que en este lugar el ala está incompleta, pero en la curvatura de los fragmentos existentes parece que no acontece antes de la transversal $\mathrm{CuP}$.
Entre la $\mathrm{RP}_{3-4}$ y la MA se observa claramente una larga vena intercalar y una o dos menores, mal definidas por la defectuosa conservación del borde apical del ala; asimismo parecen claramente visibles varias intercalares en el resto apical-superior del ala. Las celdillas entre $\mathrm{RP}_{3-4}$ y el borde posterior del ala (AP), son generalmente bastante anchas, con frecuencia mucho más anchas que altas.

Derivatio nominis: Dedicado a mi hija Diana, en cariñoso homenaje a su vocación y entusiasmo por las ciencias naturales.

\section{Discusión y conclusiones}

El ala peciolada, con dos antenodales, la celda discoidal cuadrangular, bien alejada del borde alar, el pterostigma alargado, rectangular, cubriendo dos celdillas, con una venilla oblicua entre la $\mathrm{RP}_{2}$ y la $\mathrm{IR}_{2}$, y el comienzo de $\mathrm{RP}_{3-4}$ y $\mathrm{IR}_{2}$ más cerca de la celda discoidal que del nodo, demuestran que se trata de la familia Lestidae.

La ancha celda discoidal, con el lado basal más largo que el costal y mayor que un tercio del lado inferior y la amplia separación de las bases de las venas 
$\mathrm{RP}_{3-4}$ y $\mathrm{IR}_{2}$, la mitad de su distancia del arculus, lo incluyen en la subfamilia Lestinae.

El concepto del género Lestes, basado en especies actuales es válido también para las fósiles. De este modo, la nueva especie se caracteriza en el nivel genérico de Lestes por el pterostigma moderadamente largo; la $\mathrm{RP}_{2}$ que nace aproximadamente al nivel de la $3^{\mathrm{a}}$ postnodal; el espacio entre la $\mathrm{RP}_{3-4} \mathrm{y}$ MA ampliamente ensanchado hacia el borde alar, con varias venas intercalares; las proporciones del cuadrilátero y el trazado de la transversal $\mathrm{CuP}$ respecto a la MA. Esto clasifica la nueva especie dentro del género Lestes, que respecto a las especies actuales podría ser el subgénero nominado o dudosamente el subgénero Chalcolestes, como veremos más adelante.

Respecto a la comparación de Lestes dianacompteae n. sp. con las 20 especies de fósiles que hasta ahora, según la bibliografía consultada, han sido descritas dentro de este género o posteriormente atribuidas a él, a continuación se comentarán todas ellas.

Kirby (1890) y Bridges (1993) consideraron pertenecientes a Lestes las especies Agrion coloratus, A. leucosia, A. ligea y A. peisinoe, todas de Heer, 1850, mientras que Handlirsch (1908) admitía como Lestes las anteriores especies así como Agrion iris Heer, 1879 (que Kirby considera del género Coenagrion), aunque de modo ambivalente, como probable Stenolestes. El estudio de estas cinco especies demuestra que ninguna pertenece al género Lestes, lo que confirma la opinión en este sentido de Schmidt (1958) y Nel \& Paichelar (1994).

Lestes incertus Piton, 1935, según la descripción y figuras originales, completadas con las de Nel \& Paicheler (1994), muestran una celda discoidal que hace suponer que se trata en realidad de una Sympecma mejor que un Lestes.

Lestes grandis Statz, 1930, fue pasado por Schmidt (1958) al nuevo género Oligolestes, que $\mathrm{Nel} \&$ Paicheler (1994), siguiendo a Fisher, 1974, consideran de la familia Sieblosiidae.

Lestes zalesskyi, que no ha podido ser estudiado, según Nel \& Paicheler (1994) es muy dudoso que sea un Lestidae, entre otras razones porque existe contradicción entre el texto y la figura, respecto a la situación de las venas $\mathrm{IR}_{2}$ y $\mathrm{RP}_{3-4}$ con el nodo, y la supuesta forma del pterostigma "alargado"; además, está datado como Paleoceno, lo que significaría una antigüedad de 50 millones de años, lo que en todo caso lo hace incompatible con la nueva especie.

Lestes sieblosiformis Cosmovici y Pauca H, 1955, tiene 3 venillas antenodales y la celda discoidal casi cuadrada, por lo que no pertenece al género Lestes, siendo dudosa la familia.

Tres especies atribuidas al género Lestes por sus autores, ofrecen dudas porque las descripciones e ilustraciones son incompletas o erróneas, por lo que son dudosas sus características. Son Lestes arvernus Piton, 1935, L. vicina Hagen, 1858, y L. plicata Piton y Théobald, 1939 (los dos primeros admitidos como Lestes por Bridges, 1993, el último es ignorado). En el defectuoso dibujo del ala de $L$. arvernus parece vislumbrarse que la celda discoidal es rectangular; sólo se distinguen posibles venas intercalares, el origen de $\mathrm{IR}_{2} \mathrm{y} \mathrm{RP}_{3-4}$ parece muy cercano al nodo y su final en el borde alar es sospechosamente paralelo. Suponiendo que estos caracteres sean reales, es muy distinto de la nueva especie. L. vicina muestra la $2^{\mathrm{a}}$ antenodal muy separada del arculus; la celda discoidal es muy estrecha, con el borde costal muy breve; la base de las venas $\mathrm{IR}_{2}$ y $\mathrm{RP}_{3-4}$ está demasiado alejada del arculus y hay una hilera supletoria de celdas entre MP y AP, todo lo cual la separa de la nueva especie. En cuanto a L. plicata, sólo conocida por la mitad distal de un ala, muestra escasas celdas entre la bifurcación de la IR y el pterostigma; éste aparentemente es muy pequeño y estrecho; la $\mathrm{RP}_{3-4}$ parece muy curvada hacia la base $\mathrm{y}$ entre la MP y la AP hay tres hileras de celdas, todo ello muy diferente de Lestes dianacompteae.

Las restantes ocho especies son claramente Lestes y permiten una comparación más completa con la nueva especie.

Cinco especies (L. regina, L. foersteri, L. statzi, L. ceresti y L. aquisextanus) parecen tener en común tres hileras de celdas entre la MP y la AP, es decir, una supernumeraria que puede tener de 4 a más de 12 celdillas. Este carácter, que también lo tenía alguna de las especies antes comentadas, es utilizado por Nel \& Paicheler (1994) para dividir en dos grupos los Lestes fósiles, aunque reconociendo su relativo valor sistemático y filogenético. En realidad este fenómeno es más frecuente de lo que parece, incluso en especies actuales. Schmidt (1958: 5), señala que de 1008 ejemplares de 4 especies europeas de este género, ha observado en 399 individuos celdas supletorias, que podían ser de 2 a 9. Esta particularidad es relativamente frecuente en Lestes dryas pero prácticamente nula en $L$. virens. Por esta razón se da aquí un valor secundario a este carácter, aunque señalaremos que la nueva especie sólo tiene dos hileras de celdillas.

Lestes statzi Schmidt, 1950, difiere de la nueva especie claramente por su celda discoidal mucho más estrecha; el pterostigma tiene las venillas limitantes oblicuas y cubre casi tres celdillas; el campo entre la $\mathrm{RP}_{3-4}$ y la MP es mucho más paralelo.

Lestes ceresti Nel \& Papazian, 1985, es de mayor tamaño; ala de 25,2 a $28,8 \mathrm{~mm}$; pterostigma grande, de 2,7 a $3,2 \mathrm{~mm}$ y cubre $4-5$ celdillas; las celdas supernumerarias entre la MP y AP son muy numerosas (de 7 a 12); la celda discoidal es mucho más corta, con el lado basal de $1 \mathrm{~mm}$ y el inferior de $1,7 \mathrm{~mm}$.

Lestes regina Théobald, 1937, tiene el ala del mismo tamaño que en la nueva especie, pero la distancia de la base al nodo es menor, 6,6 mm; pterostigma más largo y estrecho, de $2 \mathrm{~mm}$ de longitud y $0,5 \mathrm{~mm}$ de ancho, cubriendo 3 celdillas; celda discoidal más estrecha, con el lado basal de $0,45 \mathrm{~mm}$, y más corta, 


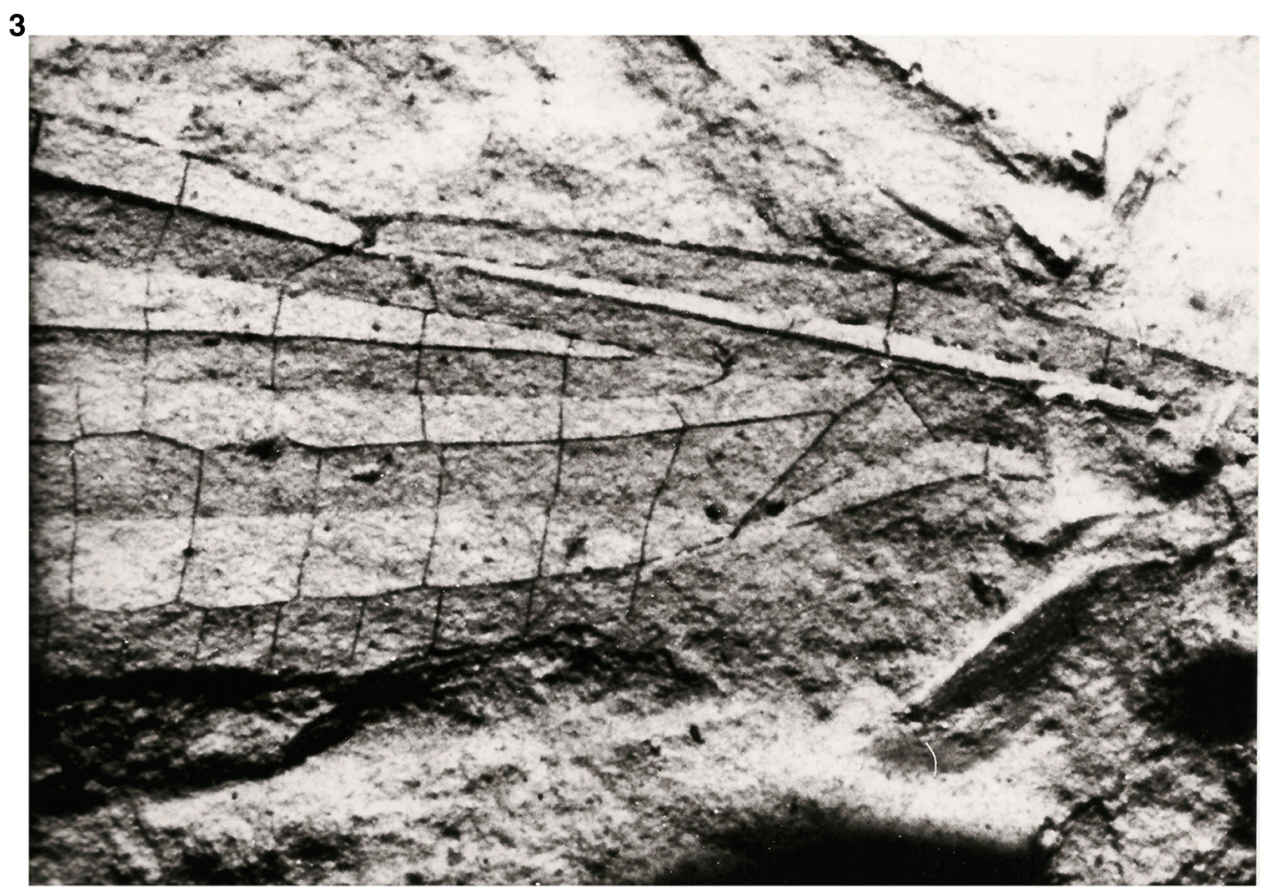

4

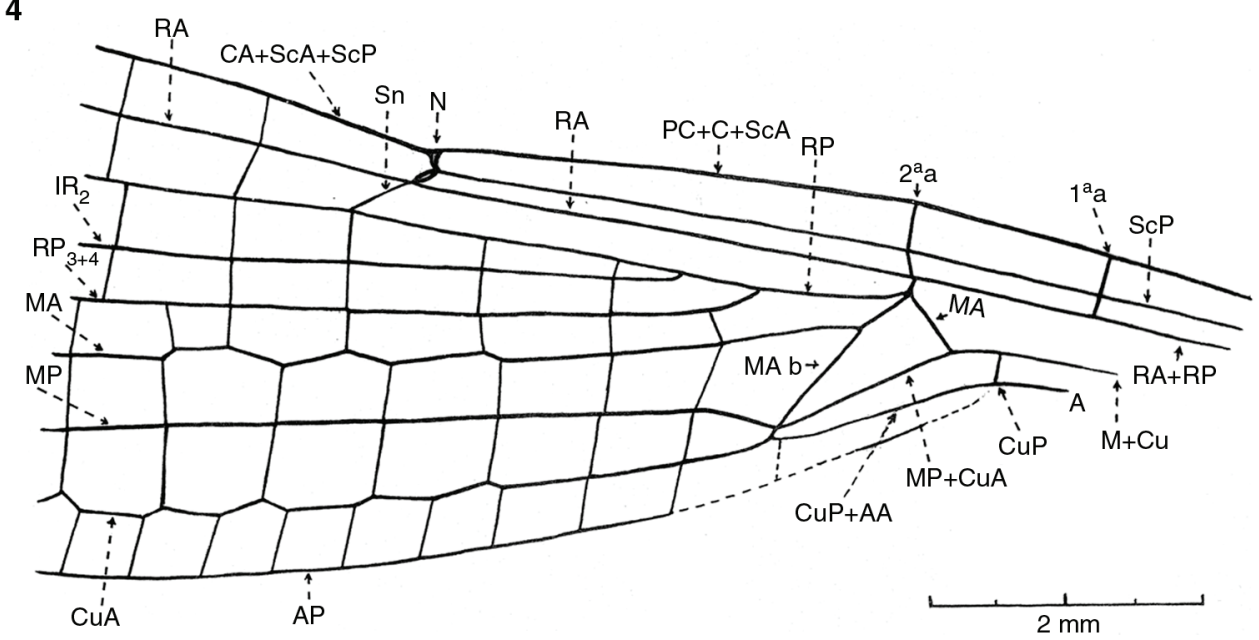

Figs. 3-4.- Lestes dianacompteae n. sp. 3) Base alar, 4) Diseño venal.

Figs. 3-4.- Lestes dianacompteae n. sp. 3) Base of the wing, 4) Venal design.

con el lado inferior de 1,2 mm. La CuP está muy cerca de la $1^{\text {a }}$ antenodal y las AA-AP se separan antes.

Lestes foersteri Hess, 1895, es ligeramente menor (ala $20 \mathrm{~mm}$ ). Según la figura de Théobald (1937: 158), efectuada sobre el holotipo y que parece ser más precisa que la de Hess, es una especie muy diferente, por la posición de la $2^{\mathrm{a}}$ antenodal, bastante anterior al arculus; la transversa CuP aparece como una prolongación del lado interno del cuadrilátero discoidal y casi al nivel de la $2^{\mathrm{a}}$ antenodal. La forma del cuadrilátero es claramente distinta, con el lado superior mucho mayor que el distal y éste similar al basal. El origen de la $\mathrm{RP}_{3-4}$ y la $\mathrm{IR}_{2}$ está mucho más cercano al arculus. Entre la MP y el borde alar hay 3 hileras de celdas, con 5 supletorias.
Lestes aquisextana Nel, 1985, es apenas mayor (ala $23 \mathrm{~mm}$ ), pero el pterostigma es más largo, 2,3 $\mathrm{mm}$, y cubre $3-4$ celdas; hay mayor número de postnodales, 14. La distancia del nodo al nacimiento de la $\mathrm{RP}_{3-4}$ es mayor, de $3,5 \mathrm{~mm}$. La distancia de la base alar al nodo también es mayor, 9,3 $\mathrm{mm}$, pero la separación del nodo al pterostigma es menor, de $9 \mathrm{~mm}$. Finalmente, entre la MP y AP hay 3 hileras de celdas.

Otra especie podría añadirse a este grupo presuntamente caracterizado por las celdillas supernumerarias entre la MP y la AP, el Lestes brisaci Nel et al. (1997), cuyo único ejemplar conocido presenta en las alas anteriores la derecha con dos celdillas intercaladas, la izquierda ninguna, pero en las alas posteriores, la derecha tiene al menos dos celdillas y la izquierda tres. 
Esto aparte, difiere de la nueva especie por ser algo menor: longitud del ala 19,2 mm, anchura $3,7 \mathrm{~mm}$; el pterostigma es proporcionalmente más estrecho, 0,4 $\mathrm{mm}$, con las venillas laterales oblicuas, cubriendo dos celdas completas; hay sólo 9 postnodales y la venilla oblicua está a una celdilla de la base de la $\mathrm{RP}_{2}$. La celda discoidal es bastante más estrecha y más corta, con el lado basal de $0,4 \mathrm{~mm}$, el distal $0,7 \mathrm{~mm}$ y el inferior $1,2 \mathrm{~mm}$.

Lestes lutzi Nel y Paicheler, 1994, es muy diferente: alas más pequeñas, $18 \mathrm{~mm}$, y proporcionalmente más estrechas, $3,3 \mathrm{~mm}$. Celda discoidal con el lado costal mayor que el basal, respectivamente 0,37 y $0,34 \mathrm{~mm}$; el campo de cuatro hileras de celdas entre la MP y la $\mathrm{RP}_{3-4}$ empieza mucho antes, poco después de la mitad de su longitud. La vena $\mathrm{RP}_{2}$ es un poco más corta y además hay menor número de celdas antes de $\mathrm{la}_{\mathrm{IR}} \mathrm{y}$ entre su origen y el pterostigma. Entre la MP y la AP hay dos celdillas suplementarias.

Lestes irenea Nel, 1987, fue descrito sobre dos ejemplares, macho y hembra, de distinta procedencia y que según su autor pueden pertenecer a dos especies distintas, por lo cual utilizaré caracteres de ambos al compararlos con la nueva especie, si bien, en el caso de confirmarse que aquéllos ejemplares son de dos especies diferentes habría no poca confusión para saber a cuál de las dos correspondería el nombre de $L$. irenea, porque su autor designó como "holotipo" un macho de la colección de Mercier, en Céreste (Nel, 1987, pág. 114), y como "alotipo" una hembra de la colección Coullet, en Barréme, pero inexplicablemente añade que ésta es el "tipo" (op. cit. págs. 113 y 116). Para aumentar la confusión, posteriormente (Nel \& Paicheler, 1994, pág. 14), se afirma lo contrario, señalando como "holotipo" el ejemplar hembra de Coullet y como "paratipo" el macho de Mercier.

Esto aparte, los dos ejemplares, aunque diferentes, son perfectamente separables de Lestes dianacompteae. En lo que se refiere al "holotipo" (de Cereste), el ala anterior es más pequeña, $18,5 \mathrm{~mm}$, pero en comparación más ancha, ya que mide $4,75 \mathrm{~mm}$ (igual que la nueva especie). Pterostigma relativamente más estrecho $(1,25 \mathrm{~mm}$ por $0,33 \mathrm{~mm})$. La distancia de la base del ala al nodo es proporcionalmente mayor que en la nueva especie, con 7,25 mm. En el cuadrilátero el lado costal es mucho más corto. En cuanto al "alotipo" (de Barrème), el ala anterior es más larga y estrecha que en el otro ejemplar, pero más parecida a Lestes dianacompteae. El cuadrilátero discal debe ser con el lado costal igualmente más corto que el basal, como en el "holotipo", pero por error la longitud del lado inferior dice que es de $2,55 \mathrm{~mm}$, cuando en realidad debe ser 1,25. El origen de las venas $I_{2}$ y $\mathrm{RP}_{3-4}$ está más próximo del arculus que en $L$. dianacompteae y sobre todo, la transversa $\mathrm{CuP}$ figura como alineada con la primera antenodal, en lugar de estar entre ésta y la segunda, como ocurre en nuestra especie.
Como conclusión de este estudio comparativo parece evidente que Lestes dianacompteae es una buena especie, distinta de todas las demás descritas y nominadas hasta ahora. Resulta difícil aventurar similitudes porque el género Lestes encierra gran número de especies (fósiles y actuales), que mezclan gran diversidad de caracteres dentro de rasgos repetitivos, muchas veces en mosaico, como se ha comentado a propósito de Lieftinck (1960). Quizá puede encontrarse alguna relación de Lestes dianacompteae con $L$. brisaci, L. irenea o L. lutzi, del Oligoceno de Francia, aunque se encuentra mayor parecido con algunos Lestes del Mioceno o tal vez Oligoceno de Francia y España, que han sido publicados sin nominarlos, por ejemplo Martínez-Delclòs \& Nel (1991), Nel \& Paicheler (1994), Peñalver et al. (1996), etc.

Respecto a la comparación con las especies actuales, ya se ha dicho anteriormente que se considera que Lestes dianacompteae puede incluirse dentro de los subgéneros Lestes s. str. o Chalcolestes, sin que pueda concretarse debido a que falta el segmento de la base alar que corresponde al punto de separación de la AA y la AP, sin certeza de si acontece después de la transversal $\mathrm{CuP}$ (Lestes) o antes (Chalcolestes), aunque la ausencia de una sinuosidad en la vena basal, muy frecuente en la inflexión de la AP en $L$. viridis, parece asegurar que no es Chalcolestes.

La especie actual que puede considerarse relativamente próxima a Lestes dianacompteae probablemente sea L. viridis (Vanderlinden, 1825), de Europa, Norte de África y Asia occidental, que tiene un aspecto general muy parecido. Sin embargo difiere por ser de mayor tamaño (ala 24-29 mm), alas más estrechas, con el espacio entre la $2^{\mathrm{a}}$ antenodal y el nodo más breve; antes de la $\mathrm{IR}_{1}$ hay menos celdas (3-4); la CuP está un poco más alejada de la celda discoidal y las venas $\mathrm{RP}_{3-4}$ y IR, salen algo más próximas al arculus. Además la inserción de la RP en el segmento anterior del arculus está en su mitad, mientras que en la nueva especie aparece junto a la celda discoidal.

\section{Agradecimientos}

A pesar del mucho tiempo transcurrido, deseo hacer constar mi agradecimiento a la entonces Directora del Museo de Geología, del Ayuntamiento de Barcelona, Dra. Alicia Masriera, y de modo especial al Conservador de Palentología del Museo, Dr. Julio GómezAlba, recientemente fallecido (2012), por su amabilidad al prestarme este fósil para su estudio (nada menos que en 1993). Asimismo por su ayuda en aquel tiempo, al Prof. D. Antonio Goy, Catedrático de Paleontología en la Facultad de Geología de la Universidad Complutense de Madrid, y al fotógrafo del mismo Departamento, D. Uly Martín, por las excelentes fotografías realizadas.

\section{Referencias}

Agustí, J. \& Roca, E., 1987. Síntesis bioestratigráfica de la fosa de la Cerdanya (Pirineos Orientales). Estudios Geológicos, 43: 521-529. http://dx.doi.org/10.3989/ egeol.87435-6619. 
Álvarez Ramis, C. \& Golpe-Posse, J. M., 1982. Sobre la Paleobiología de la cuenca de Cerdanya (depresiones pirenaicas). Boletín de la Real Sociedad Española de Historia Natural (Geología), 79: 31-44.

Anadón, P., Cabrera, L., Juliá, R., Roca, E. \& Rosell, L., 1989. Lacustrine oil-shale basins in tertiary grabens from NE Spain (Western european rift system). Palaeogeography, Palaeoclimatology, Palaeoecology, 70: 7-28. http://dx.doi.org/10.1016/0031-0182(89)90077-1.

Arillo, A. \& Sanz de Bremond, C., 1992. Nota sobre la presencia de un Tricóptero y un Odonato en el Mioceno Superior de la depresión ceretana. Boletín Geológico y Minero, 103: 16-20.

Askew, R. R., 2004. The dragonflies of Europe. (second edition). Harley Books, Colchester, Essex, 308 pp., 30 láms.

Barrón, E., 1996. Estudio tafonómico y análisis paleoecológico de la macro y microflora miocena de la cuenca de la Cerdaña. Tesis doctoral. Facultad de Ciencias Biológicas, Universidad Complutense de Madrid. (inédita). $773 \mathrm{pp}$.

Barrón, E., 1999. Estudio paleobotánico, reconstrucción paleoambiental y aspectos tafonómicos del afloramiento vallesiense de Coll de Saig (La Cerdeña, Lérida, España). Revista Española de Paleontología, ${ }^{\circ}$ extraordinario Homenaje al Prof. J. Truyols: 77-88.

Barrón, E., Rivas, M.R. \& Valle, M. F., 1996. Síntesis bibliográfica de la vegetación y clima de la península ibérica durante el Neógeno. Revista Española de Paleontología, $\mathrm{n}^{\circ}$ extraordinario, junio 1996: 225-236.

Bechly, G., 1998. The Phylogenetic Systematics of Odonata. http://members.aol.com/odonatadat/phylogeny/ system.htm.

Belyshev, B. F., 1973. [The dragonflies of Siberia (Odonata.] vol.1, part 2. Publ. House Nauka, Siberian Branch, Novosibirsk. 337-618. (en ruso).

Bridges, C. A., 1993. Catalogue of the family-group, genusgroup and species-group names of the Odonata of the world. ( $2^{\circ}$ edition). Charles A. Bridges, Urbena, Illinois.

Cabrera, L., Roca, E. \& Santanach, P., 1988. Basin formation at the end of a strike-slip fault: The Cerdanya Basin (eastern Pyrenees). Journal of the Geological Society of London, 145: 261-268.

Calvert, P. P., 1913. The fossil odonate Phenacolestes, with a discussion of the venation of the legion Podagrion Selys. Proceedings of the Academy of Natural Sciences of Philadelphia, 65: 225-272.

Calvo, J. P. Daams, R., Morales, J., López-Martínez, N., Agustí, J., Anadón, P., Armenteros, I., Cabrera, L., Civis, J., Corrochano, A., Díaz-Molina, M., Elizaga, E., Hoyos, M., Martín-Suárez, E., Martínez, J., Moissenet, E., Muñoz, A., Pérez-García, A., Pérez-González, A., Portero, J. M., Robles, F., Santisteban, C., Torres, T., Van der Meulen, A. J., Vera, J. A. \& Mein, P., 1993. Up-to-date Spanish continental Neogene synthesis and paleoclimatic interpretation. Revista de la Sociedad Geológica de España, 6: 29-40.

Carazo, F. \& Viloria, J., 1947. Noticias sobre los yacimientos fosilíferos de las margas miocenas del valle de la Cerdaña. Boletín de la Real Sociedad Española de Historia Natural, 44: 575-578.

Carpenter, F. M., 1992. Superclass Hexapoda. In: Treatise on Invertebrate Paleontology, part $R$, Arthropoda
4, vols. 3-4. Univ. Kansas and Geol. Soc. Am., Inc., Kansas. XXI et 655 pp.

Charpentier, T. von, 1841. Beschreibung eines Libellulinits aus Kroatien. Neues Jahrbuch, Mineralogie, Geognosie, Geologie, Petrefakten-kunde: 332-337, lám. 1.

Cockerell, T. D. A., 1927. A new fossil dragonfly from the Eocene of Colorado. The Entomologist, 60: 81-82.

Cosmovici, N. \& Paucá, M., 1955. Odonat fosil din Oligocenul de la Piatra Neamt, Lestes sieblosiformis n. sp. Comunicarile Academiei republicii populare romane, 5: 365-368.

Davies, A. L., 1981. A synopsis of the extant genera of the Odonata. Societas Internationalis Odonatologica, Rapid Comm., 3. Utrech, XIV-59 pp.

Davies, D. A. L. \& Tobin, P., 1984. The dragonflies of the World: A systematic list of the extant species of Odonata. Vol. 1, Zygoptera, Anisozygoptera. Soc. Int. Odonat., Rapid Comm., 3 (Suppl.), IX-127 pp.

Fraser, F. C., 1933. The fauna of British India, including Ceylon and Burma. Odonata. Vol. I. Taylor and Francis. London. 423 pp.

Fraser, F. C., 1957. A reclassification of the order Odonata. Royal Zoological Society New South Wales, Sydney. $133 \mathrm{pp}$

Hagen, H. A., 1848. Die fossilen Libellen Europas. Stettiner entomologische Zeitung, 9: 6-13.

Hagen, H. A., 1858. Zwei Libellen aus der Braunkohle von Sieblos. Palaeontographica, 5: 121-124.

Handlirsch, A., 1906-1908. Die Fossilen Insekten, und die phylogenie der rezenten formen. Engelmann, Leipzig. 1430 pp., 51 láms.

Heer, O., 1849. Die Insectenfauna der Tertiärgebilde von Oeningen und von Radoboj in Croatien. 2. Heuschrecken, Fhorfliegen, Aderflügher, Schmetterlinge und Fliegen. Engelmann, Leipzig. 269 pp., 17 láms.

Hess, W., 1895. Breitag zur Kenntnis der tertiären Agrioniden. Eine neue Lestes-Art aus dem Plattigen Steinmergel von Brunnstatt bei Mühlhausen i. E. Basel. 16 pp., 1 lám.

Kennedy, C. H., 1920. Forty-two hitherto unrecognized genera and subgenera of Zygoptera. Ohio journal of Science. 21: 83-88.

Kirby, W. F., 1890. A Synonimic Catalogue of Neuroptera Odonata, or Dragonflies. With an Appendix of Fossil Species. Gurney and Jackson, London. 202 pp.

Lieftinck, M. A., 1960. Considerations on the genus Lestes Leach, with notes on the classification and descriptions of new Indo-Australian species and larval forms (Odonata, Lestidae). Nova Guinea, zoology, 8: 127-171, 4 láms.

Margalef, R., 1957. Paleoecología del lago de la Cerdaña. Publicaciones del Instituto de Biología Aplicada, 25: 131-137.

Martín, R., 1910. Contribution à l'étude des Neuroptères de 1'Afrique. I. Les Lestes d’Afrique. Annales de la Société Entomologique de France, 79: 82-95.

Martín Closas, C., 1995. Plant Taphonomy of the la Cerdanya basin (Vallesian, Eastern Pyrenees). Geobios, 18: 287-298.

Martínez-Delclòs, X. \& Nel, A., 1991. Découverte de trois insectes fossiles dans l'Oligocène inférieur du bassin de 1'Ebre (Espagne), (Odonata, Lestidae, Aeshnidae). 
Bulletin du Muséum National d'Histoire Naturelle, París, 4me. ser., sect. C, 13: 157-165.

Menéndez Amor, J., 1955. La depresión ceretana española y sus vegetales fósiles. Característica fitopaleontológica del Neógeno de la Cerdaña española. Memorias de la Real Academia de Ciencias Exactas, Físicas y Naturales de Madrid, Ser. cienc. nat., $n^{\circ} 18.345$ pp., 52 láms.

Munz, P. A., 1919. A venational study of the suborder Zygoptera (Odonata) with keys for the identification of genera. Memoirs of the American Entomological Society, $\mathrm{n}^{\circ} 3.78$ pp., 20 láms.

Needham, J. G., 1930. A manual of the dragonflies of China. A monographic study of the chinese odonata. The Fan Memorial Institute of Biology, Peiping. 336 pp., 20 láms.

Nel, A., 1985. Sur la présence d'un Lestes Leach (1815) fossile de la lignée de Lestes regina Théobald dans les calcaires stampiens d'Aix-en-Provence (Odon., Lestidae). Entomologica Gallica, 1: 317-319.

Nel, A., 1986. Revision du genre cénozoique Stenolestes Scudder, 1895; description de deux espèces nouvelles. (Insecta, Odonata, Lestidae). Bulletin du Muséum National d'Histoire Naturelle, Section c, 8: 447-461.

Nel, A., 1987. Sur une nouvelle espèce fossile du genre Lestes Leach, 1815, du calcaire Stampien de Cereste (Alpes-de-Haute-Provence), (Odonata, Lestidae). L'Entomologiste, 43: 113-116.

Nel, A., 1991. Un nouvel odonate fossile du miocène de Bellver de Cerdana (Espagne), (Odonata, Libellulidae). Entomologica Gallica, 2: 129-130.

Nel, A., Arillo, A. \& Martínez-Delclòs, X., 1996. New fossil Odonata (Insecta) from the Upper Miocene of France and Spain (Anisoptera and Zygoptera). Neues Jahrbuch für Geologie und Palaeontologie Abhandlungen, 199: 167-219.

Nel, A., Martínez-Delclós, X., Papier, F. \& Oudard, J., 1997. New Tertiary Fossil Odonata from France (Sieblossiidae, Lestidae, Coenagrionidae, Megapodagrionidae, Libellulidae). Deutsche Entomologische Zeitschrift, 44: 231-258.

Nel, A. \& Paicheler, J. C., 1994. Les Lestoidea (Odonata, Zygoptera) fossiles; un inventaire critique. Annales de paléontologie (Vertebrates-Invertebrates), 80: 1-59.

Nel, A. \& Papazian, M., 1985. Description d'une nouvelle espèce fossile de Lestes Leach, 1815, du Stampien de Céreste (Alpes-de-Haute-Provence) (Odon. Lestidae). Entomologica Gallica, 1: 275-279.

Nel, A. \& Papazian, M. 1986. Sur une nouvelle espèce d'Odonate fossile du Stampien de Cereste (Luberón), (Odonata, Lestidae). Nouvelle Revue d'Entomologie (n.s.), 3: 227-233.

Nel, A., Petrulevicinus, J. F., Gentilini, G. \& Martínez-Delclòs, X., 2005. Phylogenetic analysis of the Cenozoic family Sieblosiidae (Insecta, Odonata), with description of new taxa from Russia, Italy and France. Geobios, 38: 219-233.

Ogg, J. G., 2004. Status of Divisions of the International Geologic Time Scale. Lethaia, 37: 183-199.

Pinhey, E., 1962. A descriptive catalogue of the Odonata of the African Continent (up to December 1959). Part I. Companhia de Diamantes de Angola, Museu do Dundo, publ. cult. $\mathrm{N}^{\circ}$ 59. Lisboa. 11-161 pp.
Pinhey, E., 1980. A revision of African Lestidae (Odonata). Occasional Papers of the National Museums and Monuments of Rhodesia, ser. B, Natural Sciences, 6: 327-479.

Piton, L., 1935. Pseudo-Néuroptères et Néuroptères des cinérites tertiaires d'Auvergne. Bulletin mensuel de la Societé Linneenne de Lyon, [1934], 78: 171-176.

Piton, L. E. \& Théobald, N., 1939. Poissons, crustacés et insectes fossiles de l'Oligocène du Puy-de-Muir (Auvergne). Mémoires de la Société des sciences de Nancy. 45 pp.

Riek, E. F. \& Kukalová-Peck, J., 1984. A new interpretation of dragonfly wing venation based upon Early Upper Carboniferous fossils from Argentina (Insecta, Odonatoidea) and basic character states in pterygote wings. Canadian Journal of Zoology, 62: 1150-1166. http://dx.doi.org/10.1139/z84-166.

Ris, F., 1921. The Odonata or Dragonflies of South Africa. Annals of the South African Museum, 18: 245-445, láms. 5-12.

Rodríguez Mellado, M ${ }^{\mathrm{a}}$ T. \& Menéndez Amor, J., 1948. Microflora fósil de Bellver. Boletín de la Real Sociedad Española de Historia Natural, 46: 69-85.

Scudder, S. H., 1985. The Miocene Insect-Fauna of Oeningen, Baden. Geological Magazine, (n.s.), 2: 116-122, 1 lám.

Schmidt, Er., 1929. Die Tierwelt Mitteleuropas (4), 7, Ordnung Libellen, Odonata. Leipzig. 66 pp.

Schmidt, Er., 1951. The Odonata of Madagascar. Zygoptera. Mémoires de l'Institut scientifique de Madagascar, 6A: $115-283$.

Schmidt, E., 1958. Bemerkungen über Lestiden III (Odonata). Uber Oligolestes grandis (Statz, 1935) und eine neue Lestes Art aus dem Mittel-Oligozän von Rott im Siebengebirge. Decheniana, 111(1): 1-7, 1 lám.

Sélys-Longchamps, M. E., 1862. Synopsis des Agrionines. Seconde légion: Lestes. Bulletin de l'Académie royal de Belgique, (2), 13: 288-338.

Sélys-Longchamps, M. E., 1883. Les Odonates du Japon. Annales de la Société entomologique de Belgique, 27: 82-143.

Sélys-Longhamps, M. E. \& Hagen, H. A., 1850. Revue des Odonates ou Libellules d'Europe. Roret, París. XXII408 pp., 11 láms.

Statz, G., 1930. Drei neue Insektenarten aus dem Tertiär von Rott am Siebengebirge. Wissenschaftlichen Mitteilungen des Vereins Natur- und Heimatkunde in Köln, 1: 10-14.

Théobald, N., 1937. Les insectes fossiles des terrains oligocènes de France. Mémoires de la Sociéte des Sciences de Nancy (Thèse Fac. Scienc. Univ. Nancy, $n^{\circ}$ 57). 473 pp., 29 láms.

Vera, J. A. (editor), 2004. Geología de España. SGE-IGME, Madrid. 890 pp.

Villalta, J. F. \& Crusafont, M., 1944. La flora miocénica de la depresión de Bellver. Ilerda, Lérida, 3 (2): 339-353, 10 láms.

Watson, J. A. L., Theischinger, G.\& Abbey, H. M. 1991. The Australian Dragonflies. CSIRO, Camberra and Melbourne. 278 pp., 24 láms. 